\title{
Nonsyndromic Craniosynostosis
}

\author{
Rebecca M. Garza, M.D. ${ }^{1}$ Rohit K. Khosla, M.D. ${ }^{1}$ \\ ${ }^{1}$ Division of Plastic and Reconstructive Surgery, Stanford University, \\ Stanford, California \\ Semin Plast Surg 2012;26:53-63. \begin{abstract}
M.D., Division of Plastic and Reconstructive Surgery, Stanford University, 770 Welch Road, Suite 400, Stanford, CA 94304 (e-mail: rkhosla@stanford.edu).
\end{abstract} \\ Address for correspondence and reprint requests Rohit K. Khosla,
}

\begin{abstract}
Nonsyndromic craniosynostosis is more commonly encountered than syndromic cases in pediatric craniofacial surgery. Affected children display characteristic phenotypes according to the suture or sutures involved. Restricted normal growth of the skull can lead to increased intracranial pressure and changes in brain morphology, which in turn may contribute to neurocognitive deficiency. Management has primarily focused on surgical correction of fused sutures prior to 12 months of age to optimize correction of the deformity and to ameliorate the effects of increased intracranial pressure. However, emphasis has recently shifted to better understanding the pathogenesis of neurocognitive impairment observed in these children, along with genetic mutations that contribute to premature suture fusion. Such understanding will provide opportunities

Keywords

- nonsyndromic craniosynostosis

- craniosynostosis

- cranial suture

- neurocognitive development for earlier and more specific neurocognitive interventions and for the development of targeted genetic therapy to prevent pathologic suture fusion.

The authors review the common types of nonsyndromic craniosynostosis and the epidemiological, genetic, and neurodevelopmental details that are currently known from the literature. In addition, they present the rationale for surgical correction, offer suggestions for timing of intervention, and present some nuances of techniques that they find important in producing consistent results.
\end{abstract}

Craniosynostosis, defined as premature fusion of cranial sutures, was first described in 1830 by Otto. ${ }^{1}$ In 1851 Virchow created a classification system for the types of skull deformity observed in craniosynostosis and made the important observation that premature suture fusion resulted in compensatory growth in other areas of the skull. ${ }^{2}$ Since Virchow, multiple theories have been proposed to explain the pathogenesis of abnormal suture fusion, with recent studies focusing on genetic regulation. Today, craniosynostosis is classified as involving a single suture versus multiple sutures and as either syndromic or nonsyndromic. Unlike the syndromic type, nonsyndromic synostosis is not associated with other dysmorphisms of the face, trunk, or extremities. Furthermore, nonsyndromic craniosynostoses typically involve a single suture, the most common types being sagittal, unicoronal, bicoronal, metopic, and lambdoidal. Although rare, multiple or pan-suture nonsyndromic synostoses exist and are referred to as "complex." 3
Sagittal synostosis is the most common form of craniosynostosis and comprises $45 \%$ of nonsyndromic cases. ${ }^{4}$ Sagittal suture fusion results in a boat-shaped deformity of the skull, termed scaphocephaly, with growth restriction in width and compensatory excessive growth in calvarial length in the anterior to posterior direction (-Fig. 1). This growth pattern leads to varying degrees of frontal bossing and occipital coning. 3,5

Unicoronal synostosis is involved in $\sim 25 \%$ of nonsyndromic cases. ${ }^{6}$ It is characterized by anterior plagiocephaly, with ipsilateral flattening of the forehead on the affected side and contralateral bulging of the frontoparietal skull (-Fig. 2). ${ }^{5}$ The growth restriction of the forehead in unicoronal synostosis results in a hallmark "facial twist." It is believed that the compensatory pressure of the ipsilateral temporal lobe pushes the maxilla forward causing a rotation of the midface. This is characterized by displacement of the ipsilateral zygoma forward as well as rotation of the maxilla so that
Issue Theme Craniofacial Surgery; Guest Editor, Edward P. Buchanan, M.D.
Copyright (C) 2012 by Thieme Medical Publishers, Inc., 333 Seventh Avenue, New York, NY 10001, USA. Tel: +1(212) 584-4662.
DOI http://dx.doi.org/ 10.1055/s-0032-1320063. ISSN 1535-2188. 

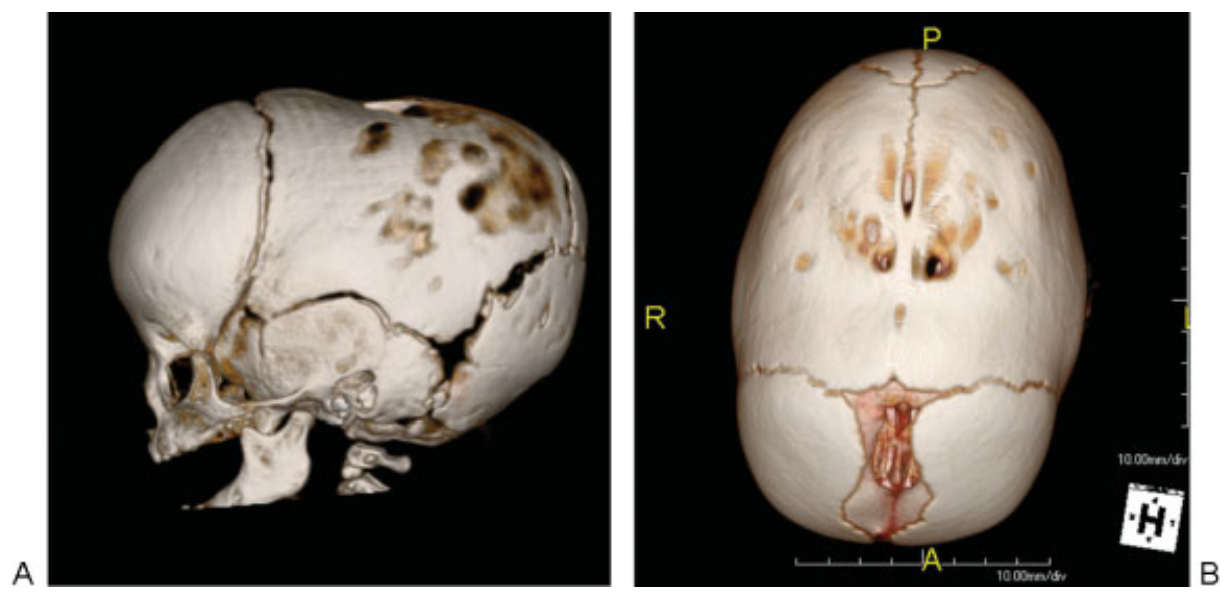

Figure 1 Three-dimensional computed tomography reconstructions of sagittal synostosis. (A) Lateral view demonstrating scaphocephaly and saddle deformity of the skull. (B) Vertex view (forehead is oriented downward) demonstrating a partially fused sagittal suture. Classic parietal and occipital narrowing is apparent.

the nasal tip is deviated to the contralateral side (-Fig. 2). In contrast, bilateral coronal fusion produces brachycephaly, skull shortening in the anterior to posterior dimension, and turricephaly, skull lengthening in a cranial to caudal dimen$\operatorname{sion}(\mathbf{- F i g} \cdot \mathbf{3})^{3}$

Metopic synostosis also occurs in $\sim 25 \%$ of nonsyndromic cases according to recent epidemiologic studies. ${ }^{6}$ Resultant trigonocephaly is characterized by a triangular-shaped forehead with bifrontal and bitemporal narrowing and parietal and occipital prominence (-Fig. 4). This also produces an appearance of hypotelorism and a low nasal dorsum with epicanthal folds. ${ }^{3}$

The rarest type of nonsyndromic craniosynostosis, unilateral lambdoidal synostosis, is characterized by occipital dysmorphism. ${ }^{3}$ The resultant phenotype has hallmark findings of an ipsilateral mastoid bulge, thickened ridging of the affected lambdoid suture, and tilt of the occipital skull base with the affected side shifted downward (-Fig. 5). ${ }^{7}$ Inferior displacement of the skull base pulls the external auditory canal downward on the affected side. The entire ear is displaced inferiorly, a clinical finding that is more reliable in differentiating positional plagiocephaly and lambdoidal synostosis than anterior or posterior displacement of the ear. ${ }^{7}$ Constriction in the region of the lambdoid suture causes a compensatory bulge in the contralateral posterior parietal region. This gives the skull an appearance of towering obliquely when viewed from behind (-Fig. 5). Finally, a phenotype similar to that produced in cases of lambdoidal synostosis results from the newly described entity of minor suture synostosis. In these cases, posterior plagiocephaly results from fusion of either the anterior or posterior intraoccipital synchondrosis. ${ }^{8}$

\section{Epidemiology and Genetics}

Craniosynostosis occurs in 1 in 2500 births, ${ }^{5}$ with the nonsyndromic subtype present in 0.4 to 1 in 1000 births. $^{9}$ In bilateral coronal, metopic, and lambdoidal nonsyndromic craniosynostosis, there is no observed gender predilection.
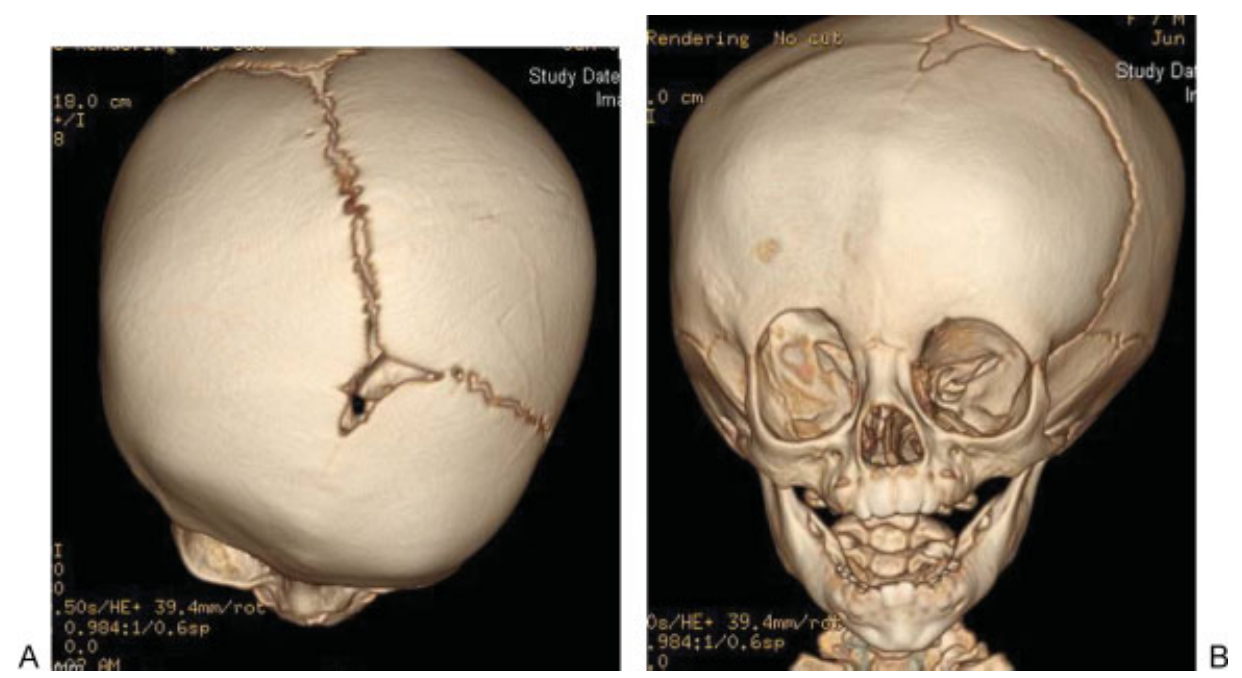

Figure 2 Three-dimensional computed tomography reconstructions of unicoronal synostosis (A) Top-down view demonstrating the unilateral forehead retrusion and anterior displacement of the zygoma on the affected side. (B) Anterior view demonstrating the periorbital deformities and maxillary rotation "facial twist," with nasal tip deviation to the contralateral side. 
A
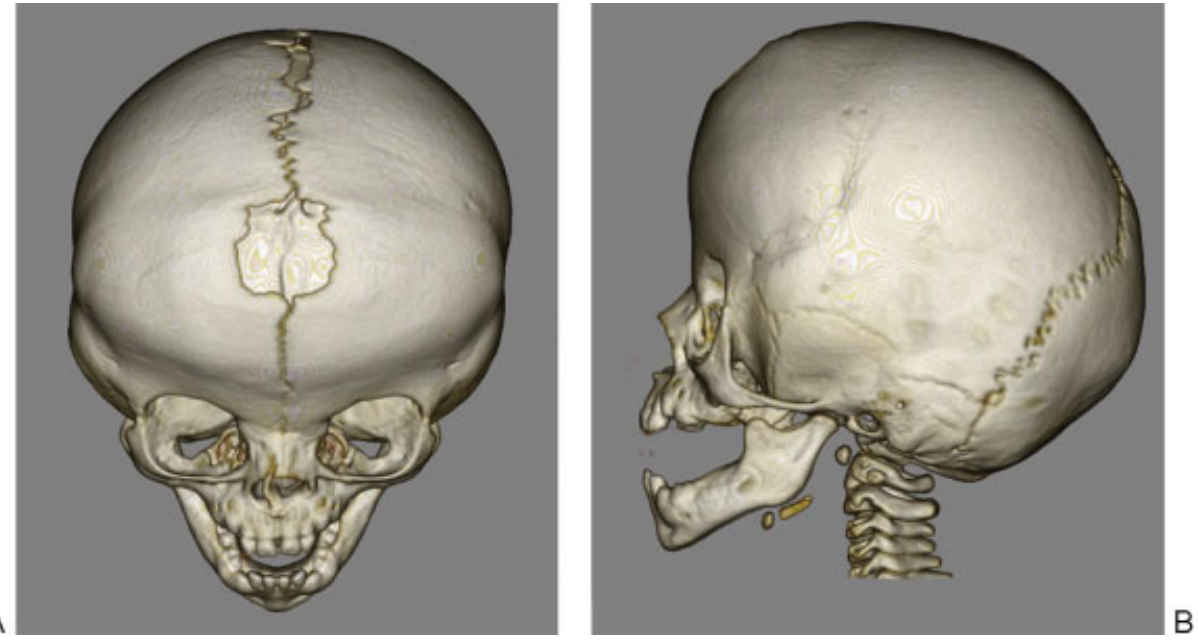

Figure 3 Three-dimensional computed tomography reconstructions of bicoronal synostosis. (A) Oblique top-down view to include the face. This demonstrates the bilateral forehead retrusion. (B) Lateral view demonstrating the typical appearance of the turribrachycephaly phenotype.
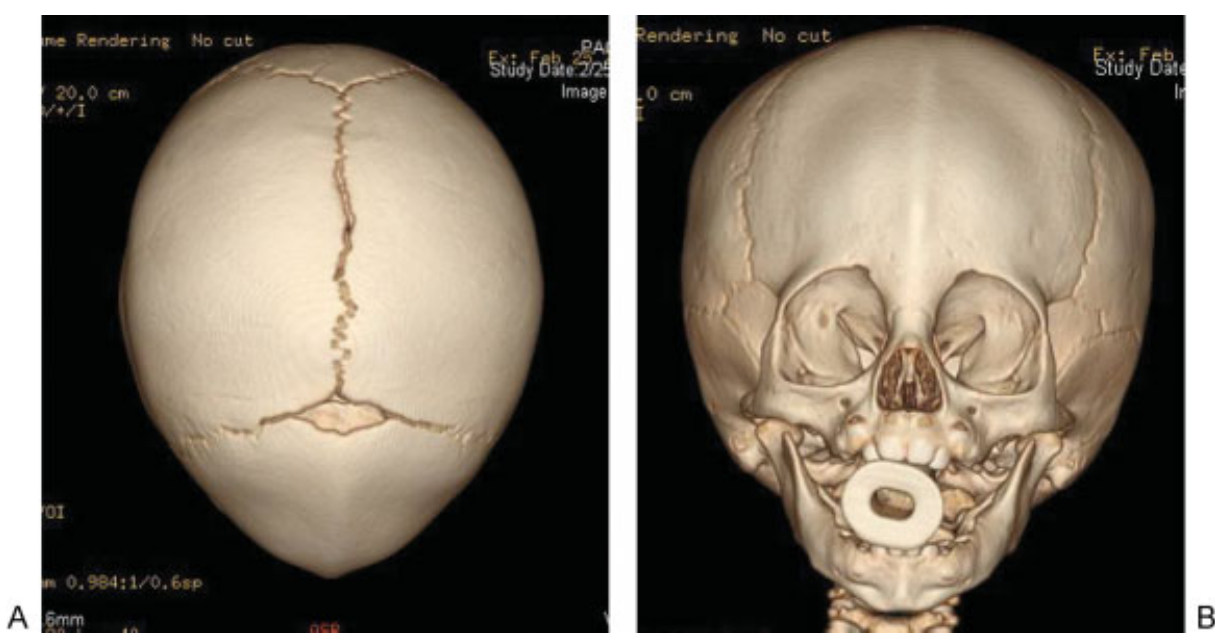

Figure 4 Three-dimensional computed tomography reconstructions of metopic synostosis. (A) Top-down view demonstrating trigonocephaly of the forehead. (B) Anterior view demonstrating the bitemporal narrowing and medialization of the superior medial orbits.
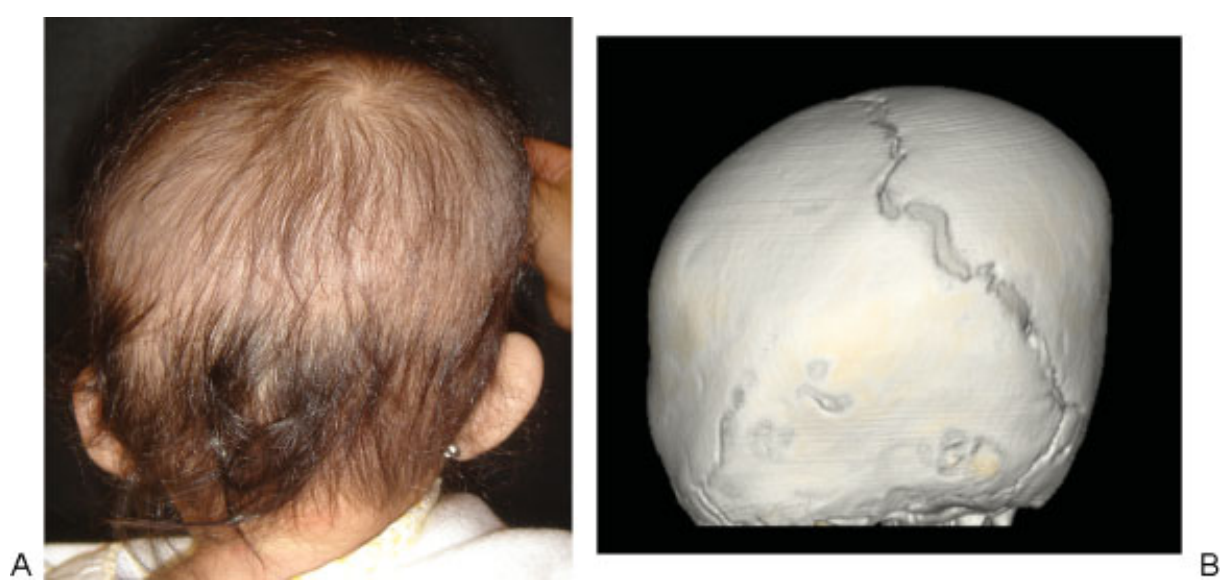

Figure 5 Unilateral lambdoid synostosis. (A) Clinical photograph of the posterior view. This shows the inferior displacement of the ear on the affected side as well as the oblique towering appearance of the skull on posterior view. (B) Three-dimensional computed tomography reconstruction (posterior view) demonstrating a partially fused left lambdoid suture. The classic mastoid bulge and tilt of the skull base is apparent. 
However, in sagittal synostosis males outnumber females in a ratio of $4: 1$; in unilateral coronal synostosis, females outnumber males in a ratio of $3: 2 .^{5}$

Several genetic mutations involving transcription factor, growth factor receptor, and cytokine expression have been identified in association with premature suture fusion in nonsyndromic craniosynostosis. Experiments in murine models have shown that fibroblast growth factor receptor gain-of-function mutations, in particular those that involve FGF2, along with mutations in transforming growth factor- $\beta$ are involved in premature suture fusion. ${ }^{10}$ Specifically, downregulation of noggin, an antagonist to bone morphogenetic proteins that belong to the TGF family and normally induce suture patency, is likely involved in pathologic suture fusion. ${ }^{11}$ Better understanding of the expression of these genes in both the dura and cranial bones, along with the timing of expression, may provide an opportunity for future targeted genetic therapy for prevention of early suture fusion.

\section{Anatomic and Neurodevelopment Consequences}

There is continued debate whether single-suture craniosynostosis leads to decreased intracranial volume, hydrocephalus, or increased intracranial pressure in affected infants. The impact of such alterations on the developing brain is also not well understood. Sgouros and colleagues investigated the effect of the type of craniosynostosis and surgical technique on intracranial volume. ${ }^{12}$ They found little difference in changes of intracranial volume among various types of craniosynostosis and discovered that procedures such as frontoorbital advancement increase intracranial volume to an above-normal level. Similarly, in a study performed by Hill et al, the authors compared intracranial volume, whole brain volume, and cerebrospinal fluid (CSF) space in control infants and infants with right unicoronal craniosynostosis ages 7 to 72 months. $^{13}$ No difference was found between the two groups, though the study was limited by its inclusion of only one type of single-suture synostosis, along with static measurements of brain morphology and exclusion of physiologic measurements such as intracranial pressure. Therefore, craniosynostosis has not been shown to be associated with low intracranial volume, perhaps because there is compensatory growth of the skull at unaffected suture sites or because bone is added to the outer, not inner, surface of the skull at the site of suture fusion. ${ }^{14}$

Similarly, no correlation between hydrocephalus and presence of craniosynostosis has been established. In cases of hydrocephalus, CSF volume may be increased with either dilation of the ventricles or dilation of pericerebral space, and such increase in volume may or may not lead to increased intracranial pressure. ${ }^{15}$ Cinalli et al performed a retrospective study of over 1700 cases of craniosynostosis, both syndromic and nonsyndromic, and evaluated for the presence of hydrocephalus in these groups. In their cohort of over 1400 infants with nonsyndromic craniosynostosis, $0.28 \%$ of patients developed hydrocephalus requiring placement of a ventriculoperitoneal shunt, all of whom were affected by complex or multiple-suture synostosis. This rate of shunting is the same as that observed in the normal population. ${ }^{16}$ In the same group, $0.6 \%$ required placement of a subduroperitoneal shunt, all of which were placed after surgical correction. Postoperative subduroperitoneal shunting was most likely needed in these cases because surgery effectively increases intracranial volume but has no effect on brain volume, thereby leading to filling of the enlarged intracranial cavity with excess CSF.

Although craniosynostosis does not seem to be associated with decreased intracranial volume or hydrocephalus, multiple studies have shown that infants with nonsyndromic and even single-suture synostoses do have an increased risk of developing intracranial hypertension. Gault and colleagues measured both intracranial volume and intracranial pressure in 66 infants with craniosynostosis. ${ }^{14}$ They used epidural sensors inserted through burr holes for pressure measurement, and intracranial volume was calculated using computed tomography (CT). The authors concluded that low volume cannot be used to predict intracranial hypertension. They also found that elevated intracranial pressure, defined as greater than $15 \mathrm{mmHg}$, was observed in $20 \%$ of children enrolled in their study. ${ }^{14}$ Most cases of intracranial hypertension were in complex or syndromic synostoses. In a similarly designed study by Renier et al, however, elevated intracranial pressure was found in $30 \%$ of children, including $14 \%$ of children with single-suture synostosis. ${ }^{17}$ Similar rates were replicated in another study by Thompson et al where prevalence of intracranial hypertension was $15 \%$ in single-suture craniosynostosis and $24 \%$ in nonsyndromic craniosynostosis, both singlesuture and complex types. ${ }^{18}$

Along with its association with intracranial hypertension, premature fusion of cranial sutures is known to alter the morphology of the underlying brain. In a series of studies by Aldridge et al, the authors showed that both cortical and subcortical structures of the central nervous system are dysmorphic in craniosynostosis. ${ }^{19}$ Specifically, studies of brain morphology in cases of sagittal and unicoronal synostosis have demonstrated that changes in brain structure are found not only in regions of the brain adjacent to the fused suture, but also distant and subcortical regions. ${ }^{20,21}$ Furthermore, these studies showed that despite surgical correction of skull shape, the brain tends to follow a growth pattern similar to that observed in patients with untreated craniosynostosis, indicating at least partially independent growth trajectories of skull and brain. ${ }^{21}$

Though some understanding exists of the interaction between premature suture fusion, intracranial volume, hydrocephalus, intracranial hypertension, and structural changes in the brain, it is ultimately the relationship of all these factors with a child's neurocognitive phenotype that must be determined. Few studies have been performed assessing the effect of increased intracranial pressure on neurocognitive development, and no consistent association has been established. ${ }^{22}$ Several recent studies have focused on nonsyndromic craniosynostosis and associated abnormalities in speech, cognition, and behavior. In a retrospective review of over 200 patients with nonsyndromic craniosynostosis, Becker et al compared speech abnormality or need for 
speech therapy, pre- and postoperative psychological test scores, school performance, and standardized test results between the general population and children with a history of craniosynostosis. ${ }^{23}$ Results were broken down according to type of affected suture. The study revealed a developmental abnormality in $47 \%$ of patients age 5 or older following corrective surgery for craniosynostosis in infancy.

A potential hypothesis for neurocognitive impairment rests on the observation that alterations in brain morphology, which persist even after surgical correction, may be responsible for observed problems with attention, language, information processing, and visual spatial skills. ${ }^{24}$ Kapp-Simon et al pose several hypotheses explaining how suture fusion may lead to observed cognitive outcomes. ${ }^{24}$ For example, sagittal synostosis seems to be associated with speech and language impairment, and the authors hypothesize that scaphocephaly may be associated with alterations in the occipital and parietal brain as well as the dorsolateral prefrontal cortex, areas known to be involved in language development, processing, and production. ${ }^{24}$ They also postulate that behavioral problems observed in children affected by metopic synostosis may be due to dysmorphism of the frontal lobes. ${ }^{24}$

The proposed hypotheses are supported by individual studies of isolated sagittal and metopic synostosis. In a study of 76 children with isolated sagittal synostosis, Shipster et al found that $37 \%$ of their cohort displayed speech, language, or cognitive impairment, a rate over 6 times that observed in the normal population. ${ }^{25}$ Additionally, they found that the vast majority of speech impairments observed could not be accounted for by generalized cognitive impairment and that expressive type of impairments were most common. Finally, the authors asserted that surgical correction of craniosynostosis does not prevent impairment or improve cognitive function. Their study was limited by small sample size, and this made drawing conclusions regarding effect of intracranial hypertension or timing of surgical intervention on developmental outcomes impossible.

Studies by both Bottero and Di Rocco et al showed that children with isolated metopic synostosis display increased rates of cognitive and behavioral problems, supported by their increased need for psychological treatment and higher incidence of attention deficit disorder. ${ }^{26,27}$ However, no direct association between severity of trigonocephaly and degree of cognitive or behavioral impairment, measured by performance on standardized neuropsychological assessments before and after surgery, has been established. ${ }^{28}$ Furthermore, Starr et al demonstrated that age of surgical correction of craniosynostosis does not seem to affect a child's risk of neurodevelopmental impairment or delay. ${ }^{28}$

\section{Indications and Timing for Surgery}

General indications for surgical intervention in nonsyndromic craniosynostosis include presence of cosmetic deformity and/or functional impairment, such as intracranial hypertension or optic atrophy. ${ }^{29}$ Most surgeons elect to delay surgery until a child is at least 3 months old and able to withstand the physiologic stresses of surgery, particularly bleeding. ${ }^{9}$ Still, ideal timing for surgical correction of craniosynostosis has been debated, and evidence supporting both early and late intervention (defined as surgery at greater than 1 year of life) exists. One of the most popular arguments for early surgical release is minimization of cerebral constriction. However, studies have shown that even without surgical correction, intracranial volume normalizes by age 6 months. ${ }^{12}$ Therefore, surgical correction to allow for cranial expansion would only be appropriate if performed in the first few months of life. This argument is weakened by the fact that no direct correlation between intracranial volume and intracranial pressure exists, and similarly, no link between intracranial volume and neurocognitive development has been established.

Other arguments for early intervention that were asserted by Renier et al include improved morphologic results, better mental level later in life, and equal rate of complications when compared with late intervention; ${ }^{29}$ more recent studies even cite lower complication rates with early intervention. ${ }^{30}$ Yet, there is little data available to compare the effects of early versus late surgical intervention. ${ }^{12}$ One possible benefit of later intervention is a lower rate of revision due to decreased incidence of restenosis. ${ }^{31,32}$ Still, many surgeons elect to operate when a child is between 3 and 6 months of age to take advantage of this period of rapid brain and skull growth, to provide an optimal chance for reossification of the surgical cranial defects, and to ensure ease of bone remodeling. ${ }^{5}$

There is no consensus on the optimal timing of surgical correction. The timing of surgical intervention is influenced by surgeon preference, timing of referral to a specialist, and preferred surgical technique. For example, endoscopic techniques are best performed as early as possible, preferably at age less than 3 months. Timing is critical, as the endoscopic technique requires several months of molding helmet therapy postoperatively to optimize results. In contrast, open techniques do not require postoperative helmet molding and can be done later, as the bones are surgically placed in, not molded to, the desired position. Generally, we prefer at our institution to perform parietal and occipital contouring (i.e., sagittal and lambdoid cases) between 3 and 6 months of age for the benefits described above. In contrast, we prefer to perform frontoorbital advancement or forehead contouring (i.e., coronal and metopic cases) between 8 and 12 months of age. We feel that the maintenance of the frontoorbital advancement is improved when the frontal bone and supraorbital rims are slightly more rigid. Thus, potential relapse of the correction is minimized.

\section{Preoperative Evaluation}

In addition to a thorough medical history and physical examination, ophthalmologic screening should be performed in affected infants, as visual abnormalities commonly accompany craniosynostosis. ${ }^{3}$ Imaging techniques such as plain radiographs, CT, magnetic resonance imaging, and ultrasound have been used in the diagnosis of and preoperative planning for craniosynostosis. Plain radiographs in children, however, are limited by low mineralization of the cranium and low 
sensitivity for detecting signs of increased intracranial pressure. ${ }^{33}$ It has become the standard of care, therefore, to obtain a CT scan with three-dimensional reconstruction. However, with recent concern over the ill effects of ionizing radiation, particularly during infancy, along with the associated cost of such scans, the need for CT in preoperative evaluation of patients with craniosynostosis has been questioned. Fearon et al showed in a prospective multicenter outcome study that the diagnostic accuracy of physical examination alone was $98 \%$ in cases of single sutural synostoses. ${ }^{34}$ Furthermore, the majority of surgeons poled in this study reported CT scans were not useful to them during surgery.

Preoperative imaging has utility in confirming diagnosis and is still performed at most institutions. Imaging is also indicated when the surgeon must evaluate for changes in brain parenchyma, signs of hydrocephalus and ventriculomegaly, presence of tonsillar herniation, or in preoperative planning for cases in which calvarial bone graft will be needed. ${ }^{34}$

\section{Surgical Management}

It must be stressed that there is no consensus on the optimal surgical techniques for skull reconstruction in any form of craniosynostosis. There are many techniques and modifications that have been described and/or presented. The techniques advocated are dependent on surgeon preference and experience alone, without comparative trials or agreed-upon aesthetic outcomes. This article is not meant to be inclusive of all surgical techniques, as that is not feasible within the scope of this article. The techniques described are those utilized by the senior author.

\section{Sagittal}

Surgical approaches for correction of scaphocephaly in sagittal synostosis range from synostectomy (either endoscopic or open), a Pi procedure that involves more extensive strip craniectomy for anteroposterior shortening, to near-total cranial vault reconstruction for children. ${ }^{35,36}$ There are several modifications to the original Pi procedure that have been described. Generally, greater degrees of deformity and scaphocephaly require lateral wedge, radial/frontal, and occipital osteotomies and subtotal calvarial reconstruction. ${ }^{3}$ For severe cases and for children who present later in life, a two-stage procedure may be required in which occipital deformity is corrected in a first stage and later followed by frontal reconstruction. ${ }^{35}$ However, in a recent study by Khechoyan et al, the authors found that frontal bossing spontaneously corrected by 2 years postoperatively following posterior-middle vault expansion alone if surgery was performed prior to 13 months of age, thereby eliminating the need for staged surgical correction. ${ }^{37}$

The senior author prefers to perform a modification of the Pi technique between 3 and 6 months of age (-Fig. 6). The operation is performed in prone position as an open technique. Success with this technique is dependent on multiple osteotomies and aggressive bone contouring of the temporal, parietal, and occipital regions. Multiple lateral barrel staves are performed down to the level of the squamosal sutures, as well as anterior to include an osteotomy in front of the coronal sutures to improve the temporal width (-Fig. 6). Wedge osteotomies are performed in the lambdoid bones to flatten and widen occipital bone contour (-Fig. 6). The occipital bones are in-fractured anteriorly at the skull base to allow some decrease in the anteroposterior dimension of the skull. Postoperatively, when a child lies on his or her back, the brain is pushed forward and laterally. Thus, floating the barrel staves laterally further improves parietal width.

\section{Coronal}

The correction of unicoronal and bicoronal synostosis requires a frontal reconstruction that addresses the superior and lateral periorbital skeleton as well as the forehead, classically described as frontoorbital advancement. Most surgeons advocate an open technique that is performed as a bifrontal orbital advancement. The senior author's preference is to perform forehead contouring between 8 and 12 months of age for the benefit described earlier.

A bifrontal craniotomy must be performed that can be done as a single piece or a double piece depending on the
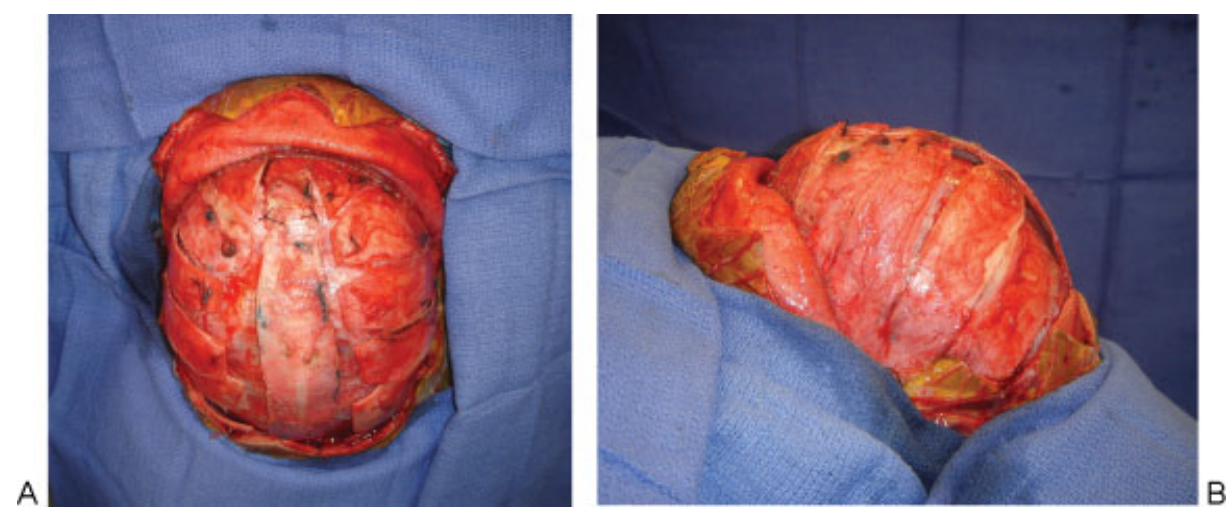

Figure 6 Intraoperative photographs of the described modified Pi procedure. (A) Posterior and vertex view with the patient in prone position. The occiput is flattened with bilateral medially based occipital wedge osteotomies. The occipital contour can be held into place with resorbable plates and screws as shown or allowed to float without fixation. (B) Lateral view with the patient in prone position. This shows the lateral barrel stave osteotomies down to the level of the squamosal sutures. The coronal suture is centered on the anteriormost barrel stave. The lambdoid suture is centered on the posteriormost barrel stave. 

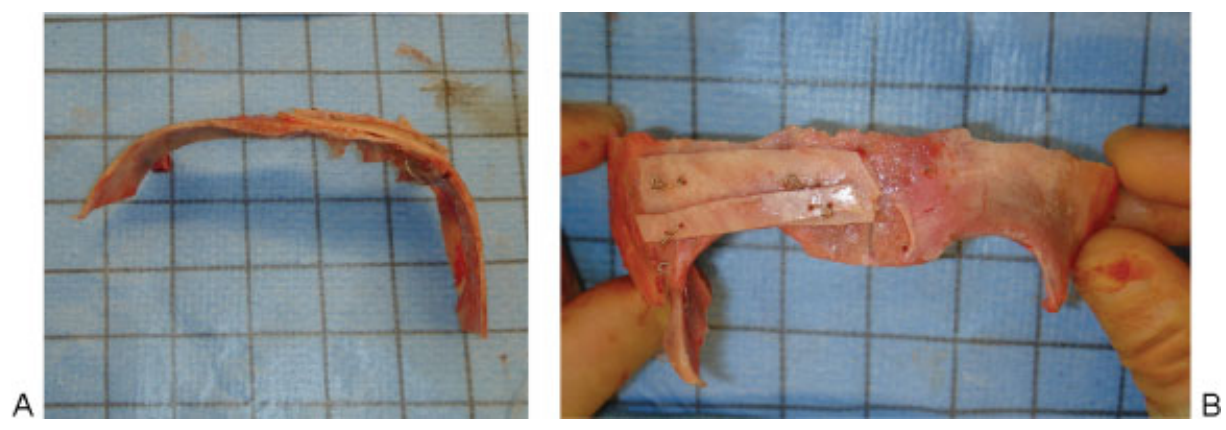

Figure 7 Reshapened frontal bandeau (ex situ) in reconstruction of unicoronal synostosis. (A) Top-down view. This shows the asymmetric design of the orbital bandeau to include a longer temporal segment on the affected side. The bandeau is bent to the desired overcorrection. Resorbable plates and screws are placed on the intracranial side at the glabella and the temporal wing on the affected side to hold the shape of the advancement and twist. (B) Anterior view. This shows the asymmetric design of the lateral orbital cuts. The osteotomy on the affected side is performed to include the entire lateral orbital rim down to the body of the zygoma, similar to a C-shaped osteotomy of the zygoma. Onlay bone grafts can be considered for additional brow projection on the affected side. However, the long-term viability and resorption of these grafts are unknown.

comfort of the neurosurgeon. Our preference is to perform the bifrontal craniotomy as a single piece, as this avoids a central frontal cranial defect. The frontal bone will need to be bent and contoured to the newly reconstructed frontal bandeau prior to replacement. The frontal bandeau is removed as a single unit.

In unicoronal cases, the frontal bandeau is cut to bring the entire lateral orbital rim on the affected side, similar in orientation to a "C-shaped" osteotomy of the zygoma ( - Fig. 7). It is believed that this prevents a bony step-off on the zygomatic frontal buttress after the advancement is performed. The bandeau is weakened in the glabellar region to bring the affected side forward. There are three pivot points in the advancement of the bandeau. One pivot point is located at the unaffected zygomatic-frontal suture, the second in the glabellar region, and the third at the body of the zygoma on the affected side. These three pivot points move in different directions and allow for the three-dimensional changes that occur with advancement of the bandeau. The glabellar pivot point is stabilized with resorbable plates and screws preferably on the internal side of the bandeau. The lateral temporal wing of the bandeau is made longer on the affected side (-Fig. 7). This area is recontoured to decrease the width of the bandeau in this location, as it is widened by the advancement at the glabella. The lateral temporal wing is contoured by a closing wedge osteotomy and plate and screw stabilization. It is critical to realize that the bandeau is not only advanced, but also twisted on the affected side to optimize brow projection. This twist maneuver rotates the temporal wing of the bandeau superiorly where it is reconnected to the parietal bone (-Fig. 8). This leaves a temporal bone cranial defect. Additional posteriorly based barrel stave osteotomies are performed in the parietal bone to elevate the parietal region that is constricted by the deformity.

These techniques are modified for treatment of bicoronal synostosis. The lateral orbital rims and C-shaped osteotomies are performed on both sides, as both lateral orbital rims need to be brought forward. The lateral temporal wings of the bandeau are extended bilaterally. The lateral closing wedge osteotomies and stabilization are performed on both sides as well. The glabellar region does not typically require recontouring in bicoronal cases. Therefore, there are only two main pivot points, one at each body of the zygoma. The advancement and twist maneuver is required on both sides to achieve improved brow projection of the bandeau ( $\mathbf{- F i g . ~ 9 ) . ~ F r o n t a l ~}$ bone recontouring is also necessary, similar to the unicoronal technique described.

\section{Metopic}

Metopic craniosynostosis must be distinguished from a benign, normally fused metopic ridge. The metopic suture fuses after birth in most patients before 1 year of age, with progression of closure from nasion to anterior fontanelle. ${ }^{38}$ By contrast, patients with metopic craniosynostosis are born with a fused suture and trigonocephaly that is easily recognizable ( $\mathbf{- F i g . 1 0}$ ). They also exhibit medialization of the orbits, which gives patients a cross-eyed and

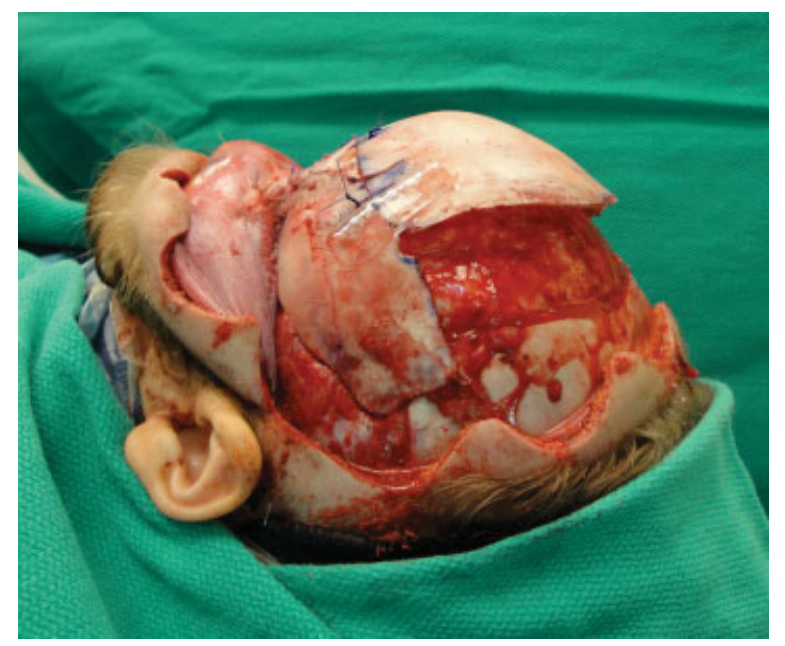

Figure 8 Lateral view of the frontal bandeau and frontal bone placed back in situ. The frontal bandeau is advanced and twisted, which rotates the temporal wing of the bandeaus superiorly. This maneuver produces enhanced brow prominence. The bifrontal bone is contoured to the reconstructed bandeau configuration and replaced as a single unit. 

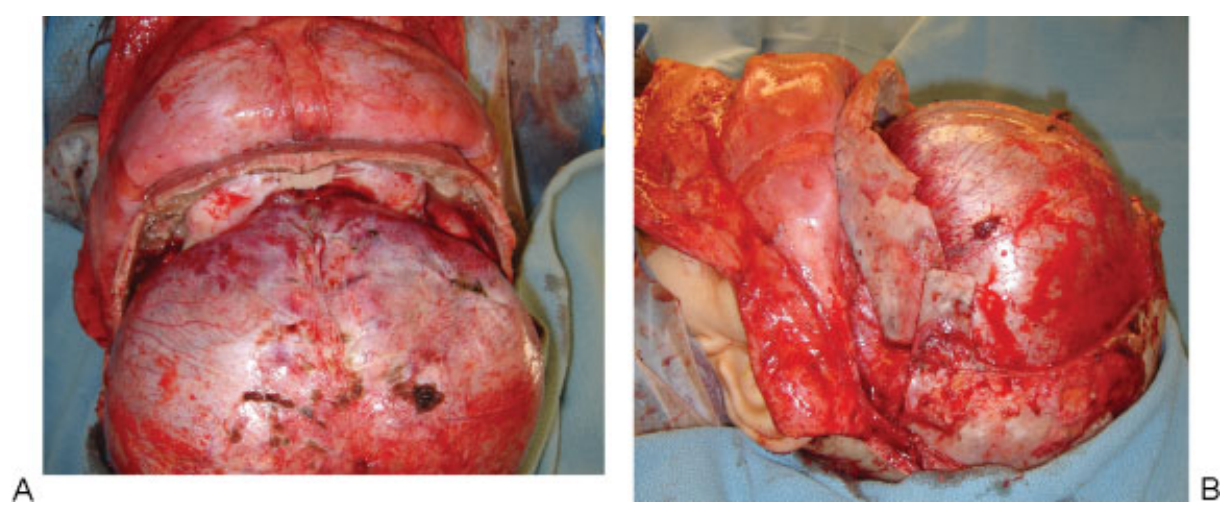

Figure 9 Reconstructed in situ appearance of the frontal bandeau. (A) Top-down view demonstrating the significant improvement in intracranial volume after appropriate advancement and twist of the frontal bandeau with fixation to the nasofrontal region and zygoma bilaterally. (B) Lateral view of the frontal bandeau showing the desired position of the temporal wings of the bandeau after the advancement and twist maneuver.

pseudo-hypoteloric appearance. A patient with benign metopic ridging will not have these hallmark features, but rather will present with a thickened, normally fused ridge several months after birth. A benign metopic ridge does not require surgical treatment.

Surgical correction for metopic craniosynostosis also requires a frontal reconstruction that addresses the superior and lateral periorbital skeleton as well as the forehead. This procedure is preferably done between 8 and 12 months of age. Most surgeons choose an open approach that allows for complete frontoorbital advancement. A bifrontal craniotomy is performed. The frontal bandeau is removed with bilateral temporal extensions. The entire lateral orbital rims are included in the bandeau as C-shaped osteotomies. Trigonocephaly results in a narrow frontal bandeau, even when it is flattened at the glabella. Therefore, it is important to widen the bandeau. This can be done by splitting the bandeau in the midline and inserting 5- to 8-mm wide bone graft (usually parietal bone) that is secured into place with resorbable plates and screws (-Fig. 11). The orbital bandeau is fixed in place with advancement and twist maneuvers to optimize brow projection.

The bifrontal bone is also too narrow in these patients. Therefore, contouring alone is usually insufficient to achieve proper alignment with the newly widened bandeau. Therefore, it is helpful to split the frontal bandeau and contour as necessary, placing it back in situ as two separate pieces. Posteriorly based barrel stave osteotomies are performed in the parietal bone extending several centimeters behind the coronal sutures to widen the vault in this area as it is also constricted by the deformity.

\section{Lambdoidal}

Correction of either unilateral or bilateral lambdoidal synostosis requires bilateral occipital and parietal reconstruction. Posterior vault reconstruction is performed between 3 and
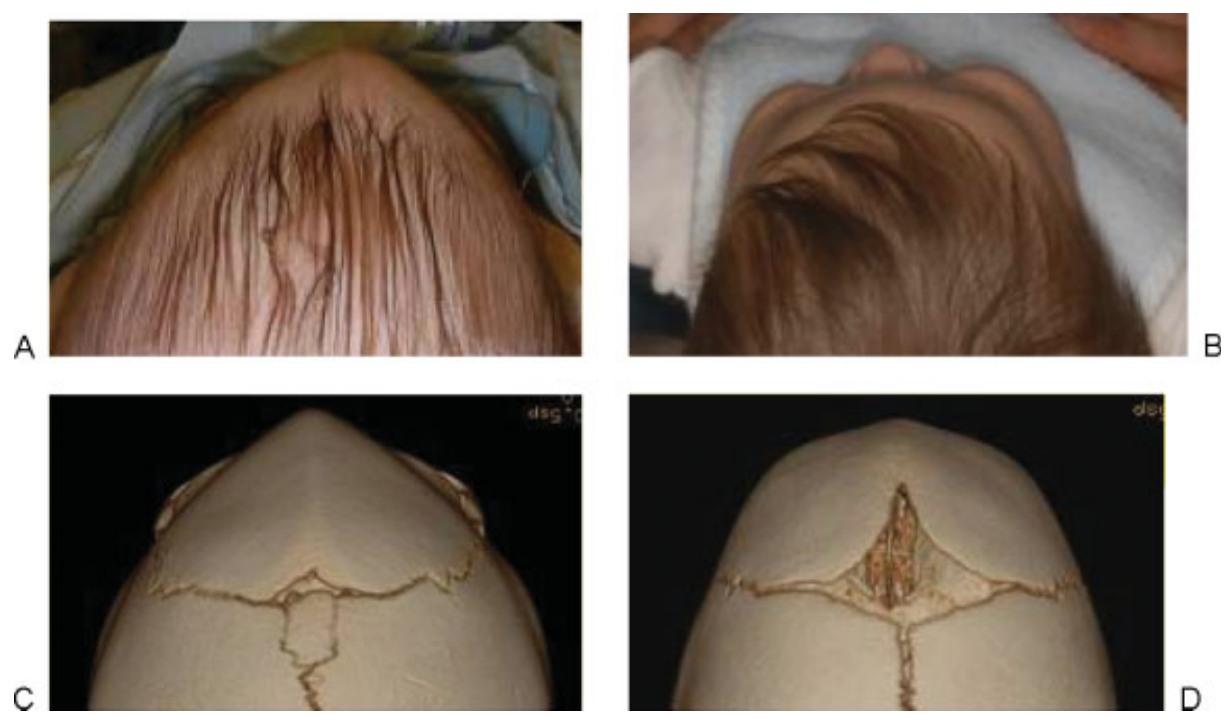

Figure 10 Forehead contour of metopic synostosis versus benign metopic ridge. (A) Typical view of forehead contour in a patient with metopic synostosis. The classic features of trigonocephaly are apparent. (B) Typical view of the forehead contour in a patient with benign metopic ridge. The forehead is normally round without trigonocephaly and normal bitemporal width. (C) Three-dimensional computed tomography reconstruction of the forehead contour in metopic synostosis. (D) Three-dimensional computed tomography reconstruction of the forehead contour in benign metopic ridge. 

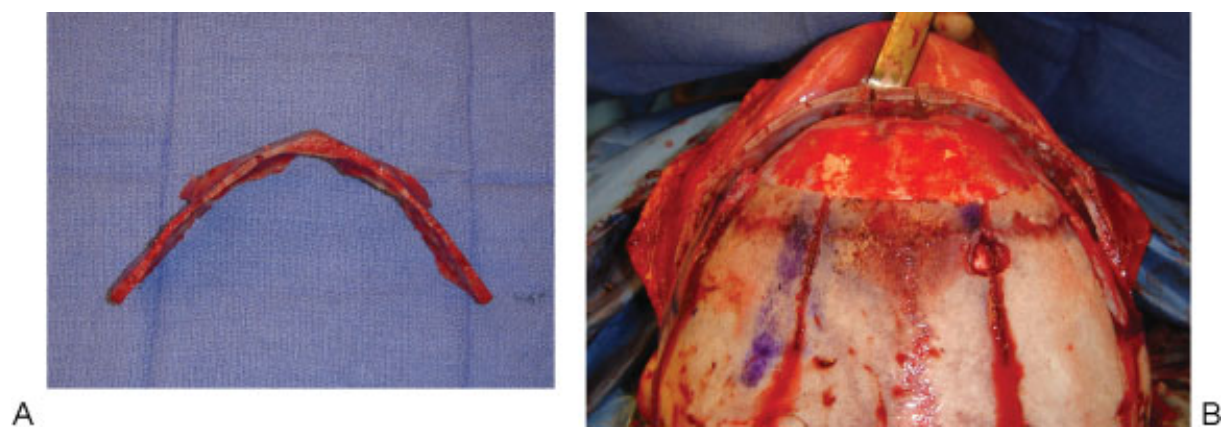

Figure 11 Frontal bandeau in metopic craniosynostosis. (A) Top-down view of the frontal bandeau ex situ prior to reshaping. (B) Top-down view of the frontal bandeau back in situ after reshaping. A central bone graft is placed in the glabellar region of the bandeau to flatten and widen the forehead contour for a more normal anatomic configuration of the forehead region. Bilateral posteriorly based barrel stave osteotomies are seen in the parietal regions, which are out-fractured to correct the narrowing of the skull in parietal regions.

6 months of age in prone position. Bilateral posterior parietaloccipital craniotomies are made. The anterior extent of the parietal cuts is made anterior to the compensatory bulging where the skull resumes normal morphology. The entire posterior parietal-occipital bone can be removed as a single piece or in two pieces with an occipital bandeau, depending on the comfort of the neurosurgeon.

The occipital bandeau is contoured and flipped to increase the volume on the constricted side. The posterior parietal bones are also flipped and oriented to achieve the most normal-appearing skull contour. Rigid internal fixation is achieved with resorbable plates and screws. Surgical cranial defects will be present and are left to reossify, as split calvarial bone grafting is not possible in patients this young.

\section{Surgical Complications and Outcomes}

Acute complications following open surgical repair of craniosynostosis include bleeding, infection, CSF leak, meningitis, stroke, and even death. Reported postoperative complications include infection, failure of reossification, contour irregularity, and need for reoperation. ${ }^{5}$ In a recent review of all cases of open craniosynostosis repair over a 12-year period, Seruya et al aimed to more clearly define both perioperative and longterm outcomes of open repair in comparison with those reported with minimally invasive techniques. ${ }^{31}$ The authors report improvement in operative time, blood loss, and average length of hospital stay when compared with rates in a similar review done in 2002. They report improvement in long-term outcomes as well, including a lower overall complication rate of 3.3\%, no deaths, improved aesthetic outcomes as measured by the Whitaker scale, and a lower reoperation rate of $10.8 \%$. Notably, patients who underwent surgical correction before 6 months of age had higher Whitaker scores, indicating worse postoperative appearance grading, and higher rates of reoperation than those infants who underwent surgical correction after 6 months of age.

A significant limitation of Seruya's study is its inclusion of all types of open repair, including less-invasive strip craniectomy and more extensive procedures for calvarial reconstruction. Not surprisingly, when the authors isolated outcomes for less-invasive open procedures, perioperative complication rates were lower. ${ }^{31}$ In another outcomes study by Panchal et al, retrospective analysis was performed on children who underwent strip craniectomy or subtotal calvarectomy at age less than or greater than 4 months. The authors compared mean cranial indices (defined as the ratio of maximal cranial width divided by maximal length multiplied by 100) which were measured by three-dimensional CT scan before surgery and again 1 year following treatment. They found that children who underwent strip craniectomy, even if performed before 4 months of age, did not achieve a normal cranial index. Those children who underwent subtotal calvarectomy, however, did achieve a cranial index that fell within the normal range if the surgery was performed before age 13 months. Importantly, the authors found no difference in outcome between infants who underwent surgery at mean age 2.9 months versus those who underwent surgery at age 7.6 months. ${ }^{39}$ Still, this study was limited by absence of follow-up beyond 1 year.

Similarly, in a study by Becker et al, the authors evaluated the long-term outcome of surgical correction for unilateral coronal craniosynostosis. ${ }^{40} \mathrm{~A}$ comparison of osseous morphology was made between patients who had reached skeletal maturity, their 1-year postoperative morphology, and the morphology of a second group of patients who had also reached skeletal maturity but who had never undergone correction of their craniosynostosis. The authors found that osseous dysmorphology did normalize when measured by CT 1 year postoperatively, but there was a tendency for reversion toward the untreated phenotype by the time skeletal maturity is reached. This tendency was also noted in a study by Fearon et al, which focused on outcomes of repair for single sutural craniosynostosis. Patients were followed for an average length of 8 years, and the authors found that although cranial indices normalized, postoperative growth was impaired in all types except lambdoidal synostosis, with greatest growth impairment perpendicular to the affected suture. ${ }^{41}$ They, like Becker et al, assert that even after surgical correction, the skull tends to follow growth patterns consistent with the original deformity. Again, like the studies described by Seruya and Panchal, Fearon et al's results demonstrated a correlation between severity of postoperative growth restriction and earlier age at time of surgical intervention. 
Despite these findings that cite limitations of early correction of craniosynostosis, there is a consensus among craniofacial surgeons, the authors included, that early surgical intervention prior to 12 months of age is preferred.

In a cohort study of over 3,000 patients who underwent surgical repair of craniosynostosis by Nguyen et al, quality outcomes assessment was performed. The authors found an overall mortality rate of $<1 \%$, complication rate of $10 \%$, and mean length of stay of 4.2 days. ${ }^{30}$ Through regression analysis, they found that patient age (1-3 years) at time of surgical intervention was the factor most significantly associated with longer length of stay, a surrogate marker for a higher complication rate. Furthermore, the authors asserted that low complication and mortality rates may be attributed to improved care provided by multidisciplinary pediatric teams, stressing the importance of these teams in caring for children with craniosynostosis.

\section{Conclusion}

Nonsyndromic craniosynostosis is commonly encountered in pediatric craniofacial surgery. Current research has focused not only on the morphologic and cosmetic consequences of these synostoses, but also associated neurocognitive impairments. Intracranial volume changes and hydrocephalus have not been associated with the nonsyndromic synostoses, but a significant proportion of affected children have been found to have both intracranial hypertension and changes in brain morphology. These children demonstrate deficiencies in language, attention, visual spatial skills, and cognitive processing that may not be corrected with surgery. Furthermore, there is a tendency for the brain and cranial vault to revert to a growth pattern consistent with the untreated phenotype even following surgical correction. Despite these limitations, surgery is recommended for both cosmetic and physiologic reasons, and most surgeons agree that early interventionless than 1 year of age-is preferred.

In the future, research must focus on attaining a better understanding of the correlation between synostosis and cognitive impairment and of genetic mutations involved. Such understanding may provide opportunities for earlier and more specific neurocognitive interventions and for the development of targeted genetic therapy for the prevention of premature suture fusion.

\section{References}

1 Otto AW. Lehrbuch der pathologischen anatomie des meuchen und der thiere. Vol. 1. Berlin: Ruecker; 1830

2 Persing JA, Jane JA, Shaffrey M. Virchow and the pathogenesis of craniosynostosis: a translation of his original work. Plast Reconstr Surg 1989;83(4):738-742

3 Marsh JM, Gurley JM, Kane AA. Nonsyndromic craniosynostosis. In: Mathes SJ, ed.Plastic Surgery. Vol. 4: Pediatric Plastic Surgery. Philadelphia, PA: Saunders Elsevier; 2006:135-164

4 Kolar JC. An epidemiological study of nonsyndromal craniosynostoses. J Craniofac Surg 2011;22(1):47-49

5 Persing JA. MOC-PS(SM) CME article: management considerations in the treatment of craniosynostosis. Plast Reconstr Surg 2008; 121(4, Suppl):1-11
6 Kolar JC. An epidemiological study of nonsyndromal craniosynostoses. J Craniofac Surg 2011;22(1):47-49

7 Ploplys EA, Hopper RA, Muzaffar AR, et al. Comparison of computed tomographic imaging measurements with clinical findings in children with unilateral lambdoid synostosis. Plast Reconstr Surg 2009;123(1):300-309

8 Sze RW, Hopper RA, Ghioni V, et al. MDCT diagnosis of the child with posterior plagiocephaly. AJR Am J Roentgenol 2005;185(5): 1342-1346

9 Shin JH, Persing JA. Nonsyndromic craniosynostosis and deformational plagiocephaly. In: Thorne $\mathrm{CH}$, Beasley RW, Aston SJ, Bartlett SP, Gurtner GC, Spear SL, eds. Grabb and Smith's Plastic Surgery. Philadelphia, PA: Lippincott Williams \& Wilkins; 2007: 226-236

10 Hunenko O, Karmacharya J, Ong G, Kirschner RE. Toward an understanding of nonsyndromic craniosynostosis: altered patterns of TGF-beta receptor and FGF receptor expression induced by intrauterine head constraint. Ann Plast Surg 2001;46(5): 546-553, discussion 553-554

11 Warren SM, Brunet LJ, Harland RM, Economides AN, Longaker MT. The BMP antagonist noggin regulates cranial suture fusion. Nature 2003;422(6932):625-629

12 Sgouros S, Hockley AD, Goldin JH, Wake MJ, Natarajan K. Intracranial volume change in craniosynostosis. J Neurosurg 1999;91(4): 617-625

13 Hill CA, Vaddi S, Moffitt A, et al. Intracranial volume and whole brain volume in infants with unicoronal craniosynostosis. Cleft Palate Craniofac J 2011;48(4):394-398

14 Gault DT, Renier D, Marchac D, Jones BM. Intracranial pressure and intracranial volume in children with craniosynostosis. Plast Reconstr Surg 1992;90(3):377-381

15 Cinalli G, Sainte-Rose C, Kollar EM, et al. Hydrocephalus and craniosynostosis. J Neurosurg 1998;88(2):209-214

16 Bondurant CP, Jimenez DF. Epidemiology of cerebrospinal fluid shunting. Pediatr Neurosurg 1995;23(5):254-258, discussion 259

17 Renier D, Sainte-Rose C, Marchac D, Hirsch JF. Intracranial pressure in craniostenosis. J Neurosurg 1982;57(3):370-377

18 Thompson DN, Harkness W, Jones B, Gonsalez S, Andar U, Hayward R. Subdural intracranial pressure monitoring in craniosynostosis: its role in surgical management. Childs Nerv Syst 1995;11(5): 269-275

19 Aldridge K, Marsh JL, Govier D, Richtsmeier JT. Central nervous system phenotypes in craniosynostosis. J Anat 2002;201(1):31-39

20 Aldridge K, Kane AA, Marsh JL, et al. Brain morphology in nonsyndromic unicoronal craniosynostosis. Anat Rec A Discov Mol Cell Evol Biol 2005;285(2):690-698

21 Aldridge K, Kane AA, Marsh JL, Yan P, Govier D, Richtsmeier JT. Relationship of brain and skull in pre- and postoperative sagittal synostosis. J Anat 2005;206(4):373-385

22 Arnaud E, Renier D, Marchac D. Prognosis for mental function in scaphocephaly. J Neurosurg 1995;83(3):476-479

23 Becker DB, Petersen JD, Kane AA, Cradock MM, Pilgram TK, Marsh JL. Speech, cognitive, and behavioral outcomes in nonsyndromic craniosynostosis. Plast Reconstr Surg 2005;116(2):400-407

24 Kapp-Simon KA, Speltz ML, Cunningham ML, Patel PK, Tomita T. Neurodevelopment of children with single suture craniosynostosis: a review. Childs Nerv Syst 2007;23(3):269-281

25 Shipster C, Hearst D, Somerville A, Stackhouse J, Hayward R, Wade A. Speech, language, and cognitive development in children with isolated sagittal synostosis. Dev Med Child Neurol 2003;45(1): 34-43

26 Bottero L, Lajeunie E, Arnaud E, Marchac D, Renier D. Functional outcome after surgery for trigonocephaly. Plast Reconstr Surg 1998;102(4):952-958, discussion 959-960

27 Kelleher MO, Murray DJ, McGillivary A, Kamel MH, Allcutt D, Earley MJ. Behavioral, developmental, and educational problems in children with nonsyndromic trigonocephaly. J Neurosurg 2006; 105(5, Suppl):382-384 
28 Starr JR, Lin HJ, Ruiz-Correa S, et al. Little evidence of association between severity of trigonocephaly and cognitive development in infants with single-suture metopic synostosis. Neurosurgery 2010;67(2):408-415, discussion 415-416

29 Renier D, Lajeunie E, Arnaud E, Marchac D. Management of craniosynostoses. Childs Nerv Syst 2000;16(10-11):645-658

30 Nguyen C, Hernandez-Boussard T, Khosla R, Curtin C. A national study on craniosynostosis surgical repair. Accepted for publication. Cleft Palate Craniofac J

31 Seruya M, Oh AK, Boyajian MJ, et al. Long-term outcomes of primary craniofacial reconstruction for craniosynostosis: a 12-year experience. Plast Reconstr Surg 2011;127(6):2397-2406

32 Wall SA, Goldin JH, Hockley AD, Wake MJ, Poole MD, Briggs M. Fronto-orbital re-operation in craniosynostosis. Br J Plast Surg 1994;47(3):180-184

33 Kotrikova B, Krempien R, Freier K, Mühling J. Diagnostic imaging in the management of craniosynostoses. Eur Radiol 2007;17(8): 1968-1978

34 Fearon JA, Singh DJ, Beals SP, Yu JC. The diagnosis and treatment of single-sutural synostoses: are computed tomographic scans necessary? Plast Reconstr Surg 2007;120(5):1327-1331

35 Fearon JA, McLaughlin EB, Kolar JC. Sagittal craniosynostosis: surgical outcomes and long-term growth. Plast Reconstr Surg 2006;117(2):532-541
36 Cohen SR, Mittermiller PA, Meltzer HS, Levy ML, Broder KW, Ozgur BM. Nonsyndromic craniosynostosis: current treatment options. In: Thaller SR, Bradley JP, Garri JI, eds. Craniofacial Surgery. New York, NY: Informa Healthcare USA, Inc.; 2008:83-102

37 Khechoyan D, Schook C, Birgfeld CB, et al. Changes in frontal morphology after single stage open posterior-middle vault expansion for sagittal craniosynostosis. 2012;129(2):504-516

38 Weinzweig J, Kirschner RE, Farley A, et al. Metopic synostosis: defining the temporal sequence of normal suture fusion and differentiating it from synostosis on the basis of computed tomography images. Plast Reconstr Surg 2003;112(5):1211-1218

39 Panchal J, Marsh JL, Park TS, Kaufman B, Pilgram T, Huang SH. Sagittal craniosynostosis outcome assessment for two methods and timings of intervention. Plast Reconstr Surg 1999;103(6): 1574-1584

40 Becker DB, Fundakowski CE, Govier DP, Deleon VB, Marsh JL, Kane AA. Long-term osseous morphologic outcome of surgically treated unilateral coronal craniosynostosis. Plast Reconstr Surg 2006; 117(3):929-935

41 Fearon JA, Ruotolo RA, Kolar JC. Single sutural craniosynostoses: surgical outcomes and long-term growth. Plast Reconstr Surg 2009;123(2):635-642 